\title{
INAA with Compton suppression: How much can the analysis of plant materials be improved?
}

\author{
M. A. Bacchi, ${ }^{1 *}$ L. G. C. Santos, ${ }^{1}$ E. A. De Nadai Fernandes, ${ }^{1}$ P. Bode, ${ }^{2}$ F. S. Tagliaferro, ${ }^{1}$ E. J. França ${ }^{1}$ \\ ${ }^{1}$ Centro de Energia Nuclear na Agricultura, Universidade de São Paulo, Caixa Postal 96, 13400-970 Piracicaba, Brazil \\ ${ }^{2}$ Interfaculty Reactor Institute, Delft University of Technology, Mekelweg 15, $2629 \mathrm{JB}$ Delft, The Netherlands
}

(Received April 13, 2006)

\begin{abstract}
The effectiveness of a Compton suppression system (CSS) for instrumental neutron activation analysis of plant materials was evaluated. Suppression factors were measured with ${ }^{137} \mathrm{Cs}$ sources. Five certified reference materials were analyzed and the detection limits calculated from both suppressed and unsuppressed spectra were compared. The CSS demonstrated to be useful for lowering the detection limits of ten out of sixteen elements tested, showing a maximum improvement factor of 3.9. The system performance was strongly influenced by the sample composition and also by the measurement conditions, indicating the importance of testing each individual sample type and analytical protocol.
\end{abstract}

\section{Introduction}

Instrumental neutron activation analysis (INAA) is known for being nearly independent on effects arising from the sample matrix. However, the sample composition still has some influence on the analytical results, since the degree of accuracy can be affected by interfering reactions or spectral interferences. Furthermore, the sample composition can also lead to acquisition of $\gamma$-ray spectra with peaks on a high background, increasing the uncertainty of peak areas and causing negative impact on the precision of results and on the detection limits. The Compton scattering of $\gamma$-rays in the detector is often the most important source of the background under low energy $\gamma$-ray peaks if measuring in the presence of high energy $\gamma$-rays. For such a case, the use of anti-Compton techniques can significantly reduce the background, improve the detection limits and also lower the measurement uncertainty.

The performance of a Compton suppression system (CSS) is usually characterized by means of suppression factors determined from spectra of either ${ }^{137} \mathrm{Cs}$ or ${ }^{60} \mathrm{Co}$ sources. The suppression factor is defined as the ratio of the peak-to-Compton ratios for suppressed and unsuppressed spectra. The effectiveness of a CSS in reducing the $\gamma$-ray spectrum background depends first of all on the equipment configuration itself and on the radionuclide being measured. Nevertheless, the sourcedetector arrangement and the source composition, i.e., the presence of other radionuclides, are also factors affecting the effectiveness of the equipment for a specific measurement. Consequently, it is difficult to estimate the improvement obtained for the determination of specific elements in a real sample, if only the suppression factor for single radionuclide sources is measured.
Since the first developments of anti-Compton techniques in the late 1960's, the use of CSS in INAA has being considered. Although COOPER and BROWNELL $^{1}$ explored the applicability of CSS for activation analysis early in 1967 , most publications are from two decades later, when dedicated systems were described $^{2,3}$ and some applications were investigated. ${ }^{4,5}$ In 1994, LANDSBERGER ${ }^{6}$ published an overview about the use of anti-Compton techniques in INAA for environmental samples.

With exceptions, ${ }^{7}$ publications about Compton suppression INAA applied to biological materials have been focused on the determination of one or a few elements. Moreover, the anti-Compton techniques have been often used ${ }^{8-10}$ in combination with epithermal neutron flux for improving the determination of elements like $\mathrm{As}, \mathrm{Cd}, \mathrm{Cu}, \mathrm{I}$ and $\mathrm{Sb}$ in the presence of high amounts of $\mathrm{Br}, \mathrm{Cl}$ and $\mathrm{Na}$. Considering such a scenario, there is still a need for studies evaluating the analysis of different matrices by Compton suppression INAA with thermal neutrons, in order to further explore its multi-element characteristics.

Here, the usefulness of a CSS for the determination of sixteen elements in plant materials was assessed. Five certified reference materials were analyzed by INAA using different measurement conditions and the detection limits calculated from both suppressed and unsuppressed spectra were compared.

\section{Experimental}

\section{Description of the Compton suppression system}

The CSS was integrated by Ortec and consists of a main HPGe detector surrounded by two secondary detectors as to provide a near $4 \pi$ configuration (Fig. 1).

\footnotetext{
*E-mail: mabacchi@cena.usp.br
} 
The HPGe detector, model GMX50220, has an n-type crystal of $257 \mathrm{~cm}^{3}$ volume, showing $55 \%$ relative efficiency and $2.26 \mathrm{keV}$ FWHM for the $1332 \mathrm{keV}{ }^{60} \mathrm{Co}$ photopeak. Both secondary detectors are $\mathrm{NaI}(\mathrm{Tl})$ crystals, a $9 " \times 9 "$ annulus and a standard $3 " \times 3 "$ plug. Compton suppression is achieved by operation in anti-coincidence mode, rejecting events occurring simultaneously in the HPGe and in any of the secondary detectors. The system was equipped with two ADC modules, allowing the concurrent acquisition of signals from the main detector as both suppressed and unsuppressed spectra.

\section{General evaluation of the system performance}

Two ${ }^{137} \mathrm{Cs}$ sources, with activities of $6 \mathrm{kBq}$ (Source 1) and $260 \mathrm{kBq}$ (Source 2) on June 2003, were employed for evaluating the system performance. To test different arrangements, sources were measured at distances between 0 and $21 \mathrm{~cm}$ from the top of the main detector, at $3 \mathrm{~cm}$ steps. The source with lower activity was used for close to detector distances (from 0 to $12 \mathrm{~cm}$ ), while the higher activity source was used from 6 to $21 \mathrm{~cm}$, allowing good counting statistics and also avoiding high counting rates. Measurements direct on the top cover of the main detector $(0 \mathrm{~cm})$ and just outside the annulus well $(9 \mathrm{~cm})$ were carried out both with and without the plug detector in place, testing its influence on the system performance. At the other positions, the plug was not used in order to evaluate the performance of the annulus detector independently.

For the measurements at 0 and $9 \mathrm{~cm}$, suppression factors were calculated for the plateau $(358-382 \mathrm{keV})$ and the edge $(475-481 \mathrm{keV})$ regions, as well as at $50 \mathrm{keV}$ intervals from 50 to $500 \mathrm{keV}$, taking $\pm 10 \mathrm{keV}$ ranges. Such procedure allowed the observation of the variation of the suppression factor with the energy (i.e., scatter angle). For all other distances the suppression factor was calculated only for the plateau region, which is the most-commonly used.

\section{Evaluation with INAA measurements}

Triplicate $300 \mathrm{mg}$ portions of five certified reference materials (Table 1) were inserted into high purity polyethylene vials and placed into aluminum rabbits. $\mathrm{NiCr}$ wires with known composition ${ }^{11}$ were used at the top and bottom of each vial for flux monitoring. Samples were irradiated in the IEA-R1m research reactor (IPEN/CNEN) at a thermal neutron flux of $10^{13} \mathrm{n} \cdot \mathrm{cm}^{-2} \cdot \mathrm{s}^{-1}$ for 8 hours.

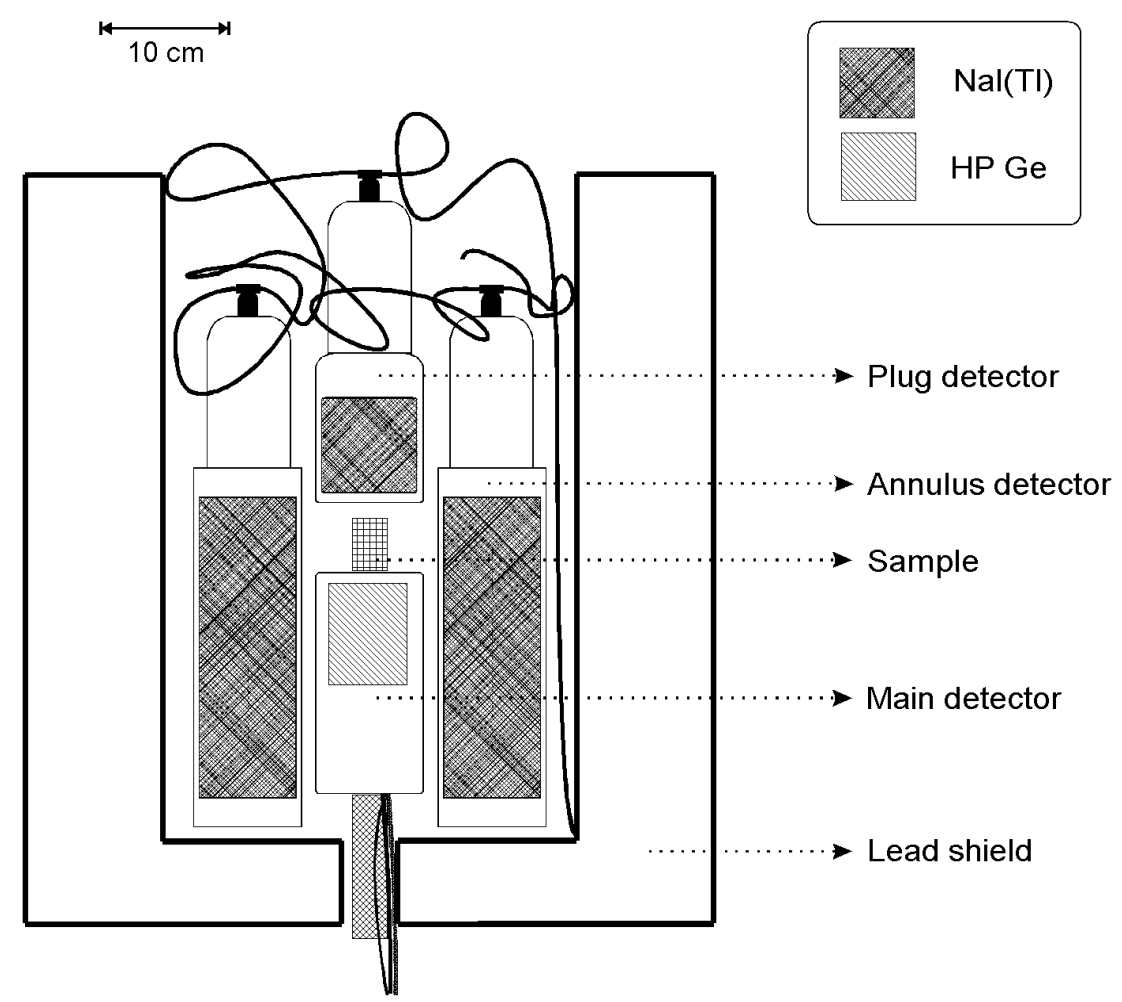

Fig. 1. Schematic representation of the CSS configuration 
Table 1. Certified reference materials used for the evaluation of the CSS effectiveness for INAA

\begin{tabular}{ccc}
\hline Material & Code & Producer \\
\hline Corn Bran & RM8433 & NIST \\
Hay Powder & V-10 & IAEA \\
Peach Leaves & SRM1547 & NIST \\
Spinach Leaves & SRM1570a & NIST \\
Tomato Leaves & SRM1573a & NIST \\
\hline
\end{tabular}

Aiming at the optimisation of measurement conditions for all radionuclides of interest, radioactivity was counted three times per sample, after decay periods of 4,8 and 18 days. Acquisition time varied from 0.5 to 2 hours, being longer for the measurements carried out long after irradiation. Sample-detector distances ranged between 0.5 and $50 \mathrm{~cm}$, set to avoid total counting rates higher than $3 \cdot 10^{3} \mathrm{cps}$. Different sample-detector arrangements were used to test the influence of distance on the performance for INAA.

Detection limits were estimated with the computer program Quantu ${ }^{12}$ from both suppressed and unsuppressed spectra, considering the uncertainty of the background in the region of the most intense photopeak of each radionuclide. Sixteen elements were evaluated, i.e., As, Br, Ca, Cd, Co, Cr, Fe, Hg, K, La, Na, Rb, Sc, $\mathrm{Se}, \mathrm{Sr}$ and $\mathrm{Zn}$.

\section{Results and discussion}

\section{Suppression factor}

For ${ }^{137} \mathrm{Cs}$, the best suppression factors were obtained with the source measured directly on the top cover of the main detector and with the plug detector in place. In such conditions, the suppression factor was $5.9 \pm 0.3$ (expanded uncertainty, $k=2$ ) for the plateau region, corresponding to a peak-to-Compton ratio of 670 for the suppressed spectrum, while for the edge region the value was $7.1 \pm 0.7$.

There was a clear variation in the suppression factors measured at different distances (Fig. 2). A linear fitting, not considering the outlier point measured at $6 \mathrm{~cm}$, indicated a reduction of about $6.5 \%$ in the suppression factor for each $10 \mathrm{~cm}$ increase in distance. For the source with higher activity, the factor measured at $6 \mathrm{~cm}$ showed a significant decrease, caused by the loss of events in the $662 \mathrm{keV}$ photopeak due to random coincidence effect, i.e., rejection of signal caused by two $\gamma$-ray emissions reaching the main detector and a secondary detector at the same time. Even for the measurements at 9 and $12 \mathrm{~cm}$, some influence of the counting rate can be noticed, since the values obtained for the source with more activity are lower. In fact, the effect of counting rate on the performance of a CSS is well-known and was previously discussed in the literature. ${ }^{13}$

The suppression factor showed to be quite variable according to the energy range selected in the Compton continuum (Fig. 3), agreeing with the variability observed by other authors. 3,14 It should be emphasized that the variation of the suppression factor with energy is strongly influenced by the configuration of the CSS regarding the position of the secondary detectors relative to the main detector and source. ${ }^{3}$ Such behavior can be observed by comparing the shape of the lines plotted for the different distances, with and without the plug detector.

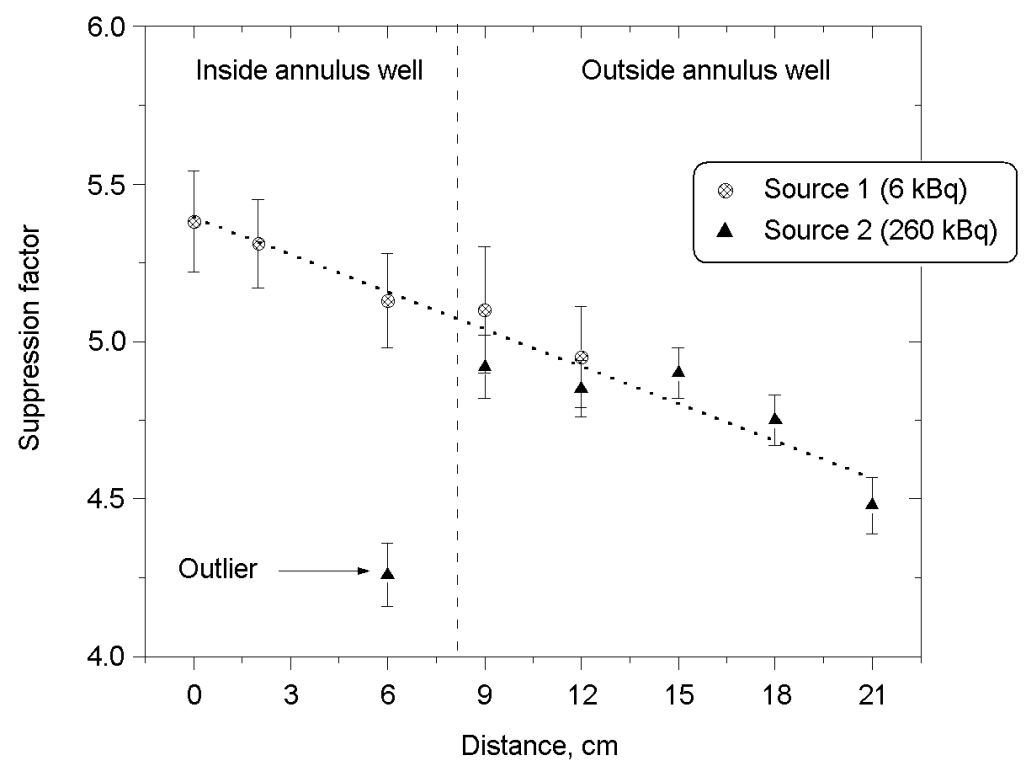

Fig. 2. Suppression factors in the plateau region, measured for ${ }^{137} \mathrm{Cs}$ sources at various distances to the main detector, without using the plug detector. Error bars represent the standard uncertainty of measurements 


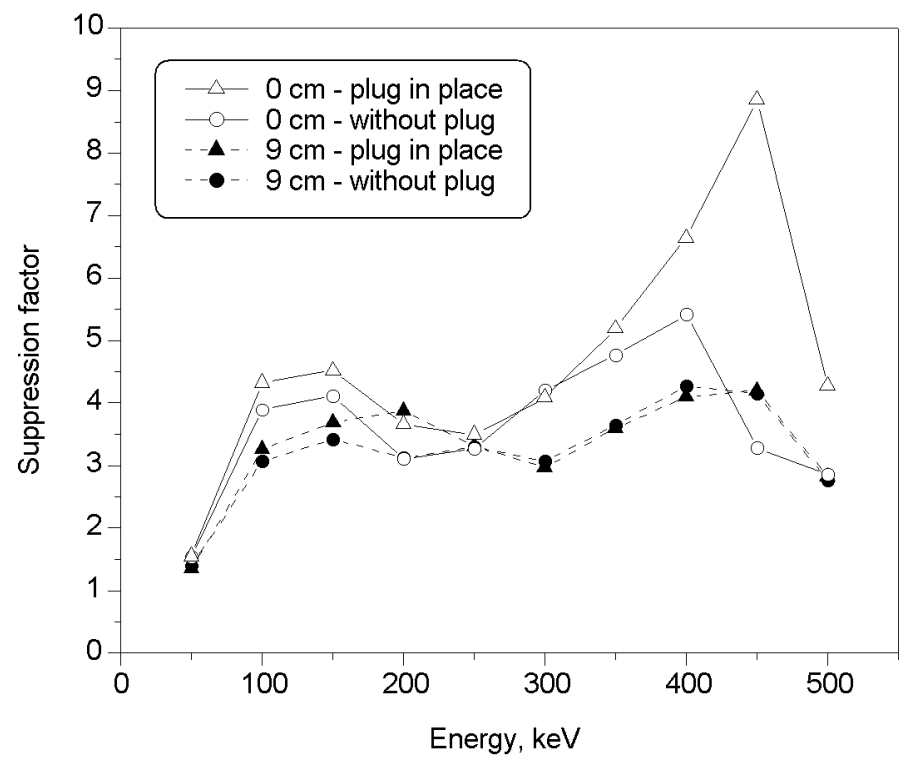

Fig. 3. Suppression factors for ${ }^{137} \mathrm{Cs}$ calculated at various energies for sources placed at the top cover of the main detector $(0 \mathrm{~cm})$ and just outside the annulus detector well $(9 \mathrm{~cm})$. Results measured with and without the plug detector

Considering its position in the CSS configuration, the plug detector is important for identifying photons scattered from the HPGe crystal with angles of more than $90^{\circ}$, which was clearly demonstrated by the suppression obtained for the energy range above $350 \mathrm{keV}$. Moreover, the plug detector had a considerable influence on the performance at positions close to the main detector, showing a very small effect at higher distances. Actually, the probability of detecting a photon scattered from the main crystal in the plug detector is strongly reduced when the plug is positioned at a high distance.

\section{Effectiveness for INAA}

The effectiveness of the CSS for INAA was evaluated by calculating improvement factors, representing the ratio of the detection limits obtained from unsuppressed and suppressed spectra. Values significantly higher than unity indicate that some improvement was obtained by the CSS, since the detection limit was reduced. On the other hand, values lower than unity demonstrate that the CSS was not useful for the determination of the specific element, at least not in the adopted experimental conditions.

Averaging the results for the five certified reference materials, the improvement factors (Fig. 4) clearly demonstrated that the CSS lowered the detection limits for $\mathrm{As}, \mathrm{Ca}, \mathrm{Cd}, \mathrm{Cr}, \mathrm{Fe}, \mathrm{Hg}, \mathrm{K}, \mathrm{Rb}, \mathrm{Sr}$ and $\mathrm{Zn}$. The best result was obtained for $\mathrm{Cd}$ with a maximum improvement factor of 3.9. In general, the observed improvement factors agree well with those predicted by
BODE, ${ }^{15}$ which are solely based on theoretical considerations.

The reduction of the detection limit is especially important for $\mathrm{As}, \mathrm{Cd}$ and $\mathrm{Hg}$, since the concentration of these elements in plants is often below the detection limits achieved by INAA. For other elements, a high improvement factor implies that the uncertainty in the determination of peak area is lowered, having a direct positive impact on the combined uncertainty of results. In some cases, significant reduction in the uncertainty of peak areas was noticed (Table 2). The relevance of such a reduction was of course dependent on the peak area itself, being important mainly for small peaks.

For $\mathrm{Br}, \mathrm{Co}, \mathrm{La}, \mathrm{Sc}$ and Se there are definitively no reasons for using the suppressed spectra. The poor performance for these elements is explained by the loss of counts in the photopeaks due to rejection of cascade events occurring in the decay of the measured radionuclides, i.e., ${ }^{82} \mathrm{Br},{ }^{60} \mathrm{Co},{ }^{140} \mathrm{La},{ }^{46} \mathrm{Sc}$ and ${ }^{75} \mathrm{Se}$. Although the determination of $\mathrm{As}$ and $\mathrm{Fe}$ was also subject to true coincidence problems, the loss of counts in the photopeaks from ${ }^{76} \mathrm{As}$ and ${ }^{59} \mathrm{Fe}$ was not as important as for the other five radionuclides. Therefore, for these two elements the background reduction was more than enough to compensate the loss of some photopeak events. For $\mathrm{Na}$, the loss of events in the $1368 \mathrm{keV}$ photopeak of ${ }^{24} \mathrm{Na}$ and the background reduction have equivalent effects in the detection limits, consequently the improvement factors were close to unity. It is important to note that the magnitude of true coincidence effects depends on the radionuclide being measured and on the source-detector arrangement. 
Table 2. Standard uncertainties (in percents) in the peak areas calculated from suppressed and unsuppressed spectra for the analysis of SRM1573a

\begin{tabular}{lccc}
\hline \multirow{2}{*}{ Element } & Energy, & \multicolumn{2}{c}{ Uncertainty } \\
\cline { 3 - 4 } & $\mathrm{keV}$ & Suppressed & Unsuppressed \\
\hline $\mathrm{Ca}$ & 1297 & 1.3 & 1.7 \\
$\mathrm{Cr}$ & 320 & 1.7 & 3.1 \\
$\mathrm{Fe}$ & 1099 & 1.4 & 2.7 \\
$\mathrm{~K}$ & 1525 & 2.6 & 3.0 \\
$\mathrm{Rb}$ & 1077 & 1.7 & 3.7 \\
$\mathrm{Sr}$ & 514 & 3.6 & 7.9 \\
$\mathrm{Zn}$ & 1116 & 1.3 & 1.7 \\
\hline
\end{tabular}

The variability noticed in Fig. 4 can be attributed mainly to the influence of the sample composition on the CSS performance. The plot of improvement factors obtained for IAEA V-10 and SRM1573a (Fig. 5) provides evidence of such an influence. These two materials represented most of the minimum and maximum values for improvement factors. Considering the lower concentrations of $\mathrm{Br}, \mathrm{K}$ and $\mathrm{Na}$, the poorest performance was expected for RM8433. However, the 13-fold higher concentration of $\mathrm{P}$ in $\mathrm{V}-10$ compared to RM8433 seems to be the main factor for the poor performance. The bremstrahlung interaction of the highenergy beta from ${ }^{32} \mathrm{P}$ results in an elevated continuum at low energies, which is not suppressed by anti-Compton spectrometry. On the other hand, the high improvement factors found for SRM1573a are due to the effective reduction of the background and photopeaks from ${ }^{82} \mathrm{Br}$, since $\mathrm{Br}$ is present at a very high concentration $\left(1300 \mathrm{mg} \cdot \mathrm{kg}^{-1}\right)$ in this material. Despite its relatively short half-life (1.47 days), ${ }^{82} \mathrm{Br}$ still remained the main source of the high background even 20 days after the irradiation.
It should be emphasized that the background spectrum originating from radionuclides with complex cascade emissions, like ${ }^{82} \mathrm{Br}$, is further reduced by CSS because of the rejection of true coincidence events. Corroborating this affirmation, regardless of the high concentration of $\mathrm{Na}$ in SRM1570a (18180 $\mathrm{mg} \cdot \mathrm{kg}^{-1}$ ), the second best performance of the CSS was noticed for SRM1547, when ${ }^{140} \mathrm{La}$ presented the main contribution for the background.

Furthermore, it is interesting to observe in Fig. 5 that opposite behavior of the improvement factors was found for $\mathrm{K}$. In fact, the contribution from ${ }^{82} \mathrm{Br}$ to the background in the ${ }^{42} \mathrm{~K}$ energy range $(1525 \mathrm{keV})$ is very low. Conversely, the Compton scatter originating from ${ }^{24} \mathrm{Na}$ is an important contribution for this spectrum region and the concentration of $\mathrm{Na}$ in $\mathrm{V}-10$ is 4-fold higher than in SRM1573a.

The effect of the sample-detector arrangement on CSS effectiveness can be demonstrated by the improvement factors obtained for $\mathrm{As}, \mathrm{Ca}$ and $\mathrm{Cd}$ in SRM1573a (Fig. 6). The variation noticed was larger than expected from the difference on suppression factors for ${ }^{137}$ Cs. This is an extreme case, since at short distances and mostly inside the annulus well, the rejection of cascade events from ${ }^{82} \mathrm{Br}$ increases drastically, resulting in extra reduction of the spectrum background. At lower degrees, variation in the improvement factors with sample-detector distance was also observed for the other materials. For $\mathrm{K}$ in the RM8433, for instance, the improvement factors were 1.3, 2.0 and 2.2 at distances of 50,16 and $8 \mathrm{~cm}$, respectively, indicating a linear behavior, which agrees with the difference in the suppression factors observed for ${ }^{137} \mathrm{Cs}$.

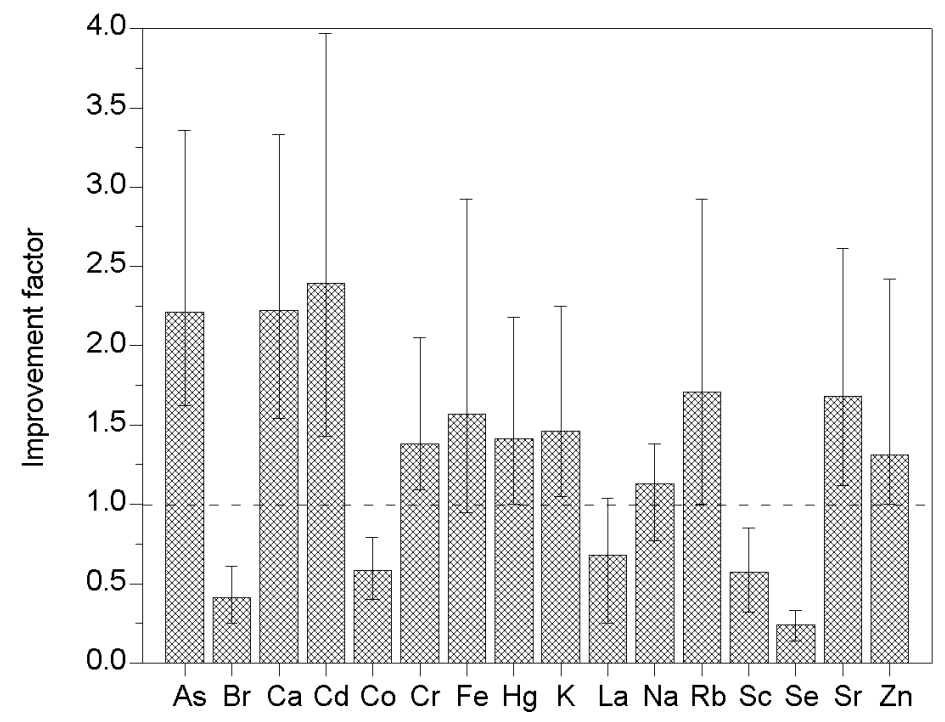

Fig. 4. Average improvement factors for the five certified reference materials, calculated from the individual detection limits. Error bars represent the maximum and minimum values 


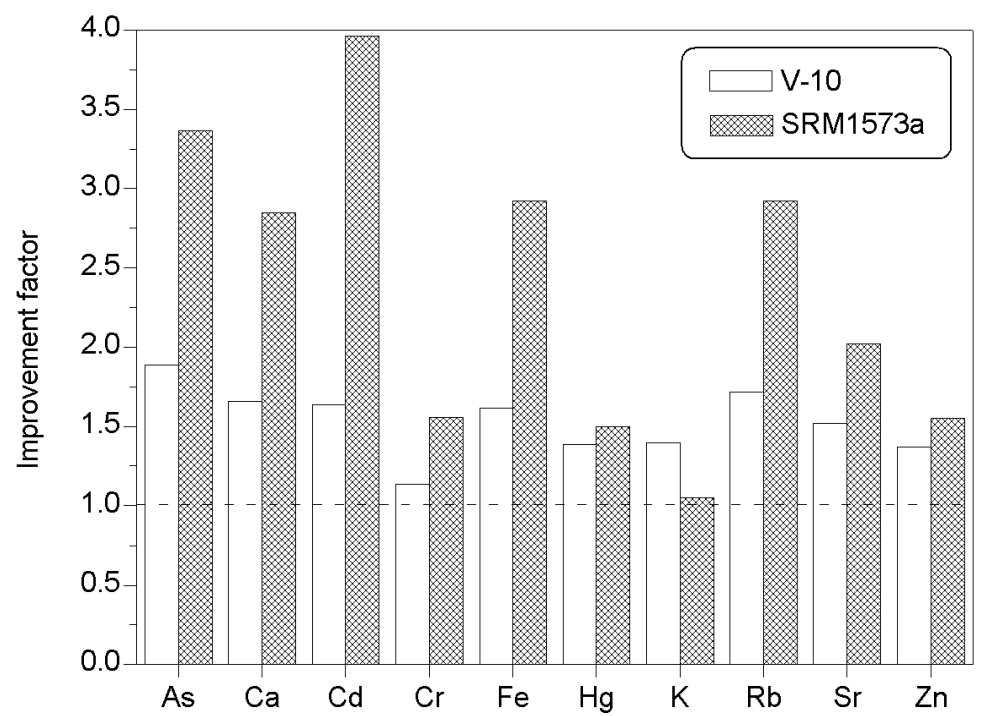

Fig. 5. Improvement factors obtained for the two reference materials showing the maximum and the minimum influence from the anti-Compton system

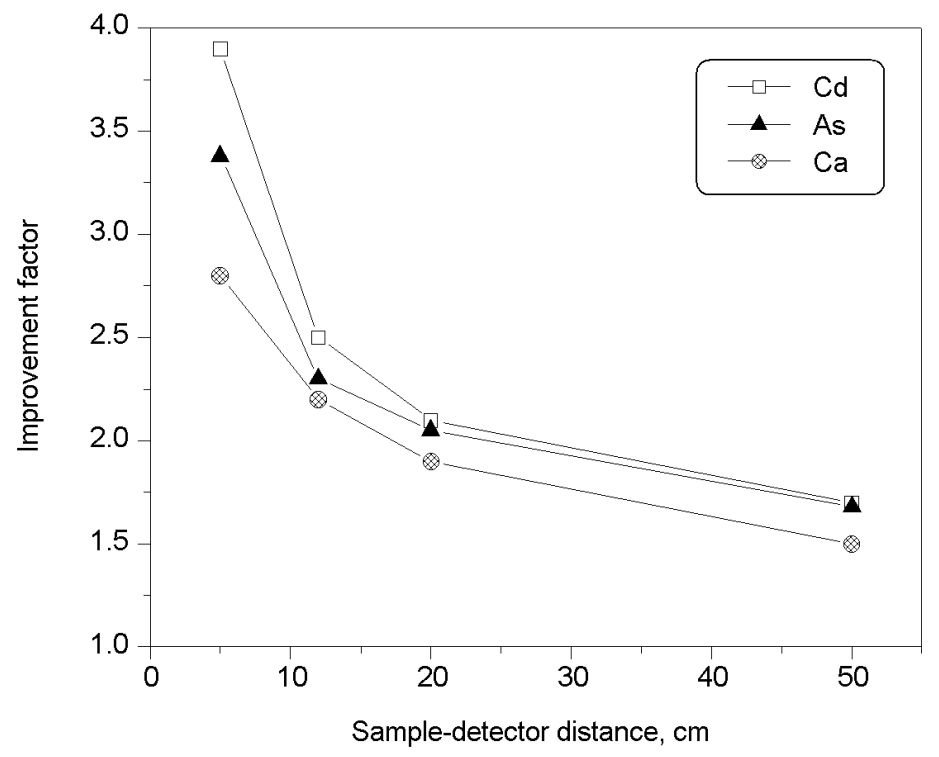

Fig. 6. Improvement factors measured at various sample-detector distances for As, Ca and Cd in SRM1573a

\section{Conclusions}

For ten out of sixteen elements evaluated, the CSS showed significant reduction in the detection limits obtained for INAA of plant materials. The results confirmed that the composition of sample is a primary factor affecting the effectiveness of a CSS for the determination of a given element. Each individual sample type should be tested in order to have a realistic estimate of the possible improvement. Even so, the occurrence of an occasional high concentration of a trace element can have a significant impact on the performance of a CSS for INAA. The sample-detector distance also influenced the performance, indicating that the measurement condition has to be carefully considered.

The authors are indebted to FAPESP (01/12141-0 and 04/05626-6), PRONEX and FNDCT/FVA, Brazilian organizations that provided financial support for this research. 


\section{References}

1. R. D. CoOper, G. L. Brownell, Nucl. Instr. Meth., A51 (1967) 72.

2. M. Petra, G, Swift, S. LANDSBERger, Nucl. Instr. Meth., A299 (1990) 85.

3. E. Mauerhofer, U. Tharun, H. O. Denschlag, R. Schmidt, J. V. KRATZ, Nucl. Instr. Meth., A371 (1996) 465.

4. M. Rossbach, R. Zeisler, J. R. W. WoitTiEZ, Biol. Trace Elem. Res., 26/27 (1990) 63.

5. S. Landsberger, S. Larson, D. Wu, Anal. Chem., 65 (1993) 1506.

6. S. LANDSBerger, J. Radioanal. Nucl. Chem., 179 (1994) 67.

7. X. LIN, C. LIERSE, W. WAHL, J. Radioanal. Nucl. Chem., 215 (1997) 169.

8. S. LANDSBERGER, D. Wu, Sci. Total Environ., 173 (1995) 323.
9. M. A. Deibel, S. Landsberger, D. Wu, W. D. Ehmann, J. Radioanal. Nucl. Chem., 217 (1997) 153.

10. Y. SERFor-ARMAH, B. J. B. NyARKo, J. HolzBecher, E. H. K. AKAho, E. K. Osae, A. Chatt, J. Radioanal. Nucl. Chem., 256 (2003) 259.

11. E. J. FrançA, E. A. N. Fernandes, M. A. BACChi, J. Radioanal. Nucl. Chem., 257 (2003) 113.

12. M. A. BaCChi, E. A. N. Fernandes, J. Radioanal. Nucl. Chem., 257 (2003) 577

13. G. P. Westphal, K. JÖStl, P. Schröder, R. LAUSTER, E. HAUSCH, Nucl. Instr. Meth., A422 (1999) 347.

14. J. PARUS, J. KierzeK, W. RAAB, D. Donohue, J. Radioanal. Nucl. Chem., 258 (2003) 123.

15. P. BoDE, J. Radioanal. Nucl. Chem., 222 (1997) 127. 